\title{
PSEUDOCOMPACTNESS PROPERTIES
}

\author{
SAMUEL BROVERMAN 1
}

\begin{abstract}
A topological extension property is a class of Tychonoff spaces $\mathcal{P}$ which is closed hereditary, closed under formation of topological products and contains all compact spaces. If $X$ is Tychonoff and $\mathscr{P}$ is an extension property, there is a space $\mathscr{P} X$ such that $X \subseteq \mathscr{P} X \subseteq \beta X, \mathscr{P} X \in \mathscr{P}$ and if $f: X \rightarrow Y$ where $Y \in \mathscr{\rho}$ then $f$ admits a continuous extension to $\mathscr{P} X$. A space $X$ is called $\mathscr{P}$-pseudocompact if $\mathscr{P} X=\beta X$. In this note it is shown that if $\mathscr{P}$ is an extension property which contains the real line (e.g., the class of realcompact spaces), $X$ is $\mathscr{P}$-pseudocompact and $Y$ is compact, then $X \times Y$ is $\mathcal{P}$-pseudocompact. An example is given of an extension property $\mathcal{P}$, a $\mathscr{P}$ pseudocompact space $X$ and a compact space $Y$ such that $X \times Y$ is not $\mathscr{P}_{\text {- }}$ pseudocompact.
\end{abstract}

1. Introduction. If $\mathcal{P}$ is a class of completely regular, Hausdorff spaces which contains all compact spaces such that $\mathscr{P}$ is closed under the formation of topological products and closed subspaces, then $\mathscr{P}$ is called a topological extension property. Such classes may also be familiar as the almost-fitting epireflective subcategories of the category of completely regular, Hausdorff spaces. If $X$ is a completely regular space and $\mathscr{P}$ is an extension property then there is a space $\mathscr{P} X$ such that $X \subseteq \mathscr{P} X \subseteq \beta X, \mathscr{P} X \in \mathcal{P}$, and every continuous map from $X$ to a space in $\mathcal{P}$ admits a continuous extension to $\mathscr{P} X$. The basic references for this material are [5] and [9]. In what follows, a space will mean a completely regular, Hausdorff space. Given an extension property $\mathscr{P}$ and a space $X$, we call $X \mathscr{P}$-pseudocompact if $\mathscr{P} X=\beta X$. The class of $\mathscr{P}$-pseudocompact spaces is denoted by $\mathscr{P}^{\prime}$. Woods [9] introduced this notion of $\mathcal{P}_{-}$ pseudocompactness and investigated the class $\mathscr{P}^{\prime}$. If $\mathscr{P}$ is the class of realcompact spaces then $\mathscr{P}^{\prime}$ is the class of pseudocompact spaces. In Theorem 2.2 of [9], it is shown that the class $\mathcal{P}^{\prime}$, for an arbitrary extension property $\mathscr{P}$, satisfies many of the properties enjoyed by the class of pseudocompact spaces. It is well known that the topological product of a pseudocompact space with a compact space is pseudocompact (see 9.14 of [4]). It is also known (see Proposition 2.5 of [8]) that a space $X$ is pseudocompact if and only if $E(X)$, the projective cover of $X$, is pseudocompact. In [9], the question is raised of whether or not the class $\mathscr{P}^{\prime}$ satisfies the above two conditions for an arbitrary

Received by the editors December 24, 1975.

AMS (MOS) subject classifications (1970). Primary 54D60, 54C20.

Key words and phrases. Stone-Čech compactification, extension property, realcompact, pseudocompact.

1 This research was partially supported by a scholarship from the National Research Council of Canada.

() American Mathematical Society 1976 
extension property $\mathcal{P}$. In this paper we show that the answer to both questions is, in general, no; however for a large class of extension properties $\mathcal{P}$ the answer to the first question is yes.

The notation will follows that of [4], and $N$ will denote the natural numbers.

\section{Pseudocompactness properties.}

2.1. ExAmple. Let $\mathcal{P}$ be the class of spaces all of whose connected components are compact. Clearly $\mathcal{P}$ is an extension property. It is also easy to see that $\mathbf{R} \in \mathcal{P}^{\prime}$ (where $\mathbf{R}$ is the real line). But $E(\mathbf{R})$ is extremally disconnected and noncompact. In particular, $E(\mathbf{R}) \in \mathcal{P}$ as $E(\mathbf{R})$ is totally disconnected, hence connected components of $E(\mathbf{R})$ are points. But by Theorem 2.2 of [9], we must have that $E(\mathbf{R}) \notin \mathcal{P}^{\prime}$ as $E(\mathbf{R})$ is noncompact. This answers the second question mentioned in the introduction.

2.2. ExAmple. In what follows, let $\mathscr{P}$ denote the class of all spaces $X$ such that every countable subset of $X$ has compact closure in $X$. Such spaces are called $\boldsymbol{\kappa}_{0}$-bounded spaces, and it is shown in [7] that $\mathscr{P}$ is an extension property and furthermore that if $X$ is a space then $\mathscr{P} X$ consists of all points in $\beta X$ which are in the closure of a countable subset of $X$. Clearly any separable space is in $\mathcal{P}^{\prime}$, hence $N \in \mathcal{P}^{\prime}$. We show that $N \times \beta D \notin \mathcal{P}^{\prime}$ where $D$ denotes the discrete space of cardinality $\boldsymbol{N}_{1}$.

Let $\{0,1\}$ denote the two-point discrete space. Let $X$ be the $\Sigma$-space of $\{0,1\}^{\aleph_{1}}$, i.e., $X$ consists of those points in $\{0,1\}^{\aleph_{1}}$ which have at most countably many coordinates equal to 1 . Then $X \in \mathcal{P}$. For each $i \in N$ define

$$
A_{i}=\left\{p \in\{0,1\}^{\aleph_{1}}:\left|\left\{k: \pi_{k}(p)=1\right\}\right| \leqslant i\right\}
$$

where $\pi_{k}$ is the $k$ th projection map from $\{0,1\}^{N_{1}}$ to $\{0,1\}$. Clearly each $A_{i}$ is compact, and if $A=\cup_{i \in N} A_{i} \subseteq X$ then $A$ is dense in $X$. Thus $A \notin \mathcal{P}^{\prime}$. For by Theorem 2.2 of [9], if $A \in \mathscr{P}^{\prime}$ then $X \in \mathscr{P}^{\prime}$, hence $X \in \mathscr{P} \cap \mathcal{P P}^{\prime}$ and $X$ must be compact, which is not true. Since $\left|A_{i}\right|=\aleph_{1}$ for every $i \in N$, we have that $A_{i}$ is the continuous image of $\beta D$. Thus $A=\cup_{i \in N} A_{i}$ is the continuous image of $N \times \beta D$. Since $A \notin \mathcal{P}^{\prime}$ we have that $N \times \beta D \notin \mathcal{P}^{\prime}$ by Theorem 2.2 of [9]. This answers question one.

2.3. TheOREM. Suppose that $\mathcal{P}$ is an extension property such that $\mathbf{R} \in \mathcal{P}$, i.e., $\mathcal{P}$ contains all realcompact spaces. Then if $X \in \mathcal{P}^{\prime}$ and $Y$ is compact then $X \times Y \in \mathcal{P P}^{\prime}$.

Proof. By 2.2(e) of [9], every member of $\mathcal{P}^{\prime}$ is pseudocompact. Let $X \in \mathcal{P}^{\prime}$ and let $Y$ be compact. Then $X \times Y$ is pseudocompact. Hence, by Theorem 2.1 of [3], $\beta(X \times Y)=\beta X \times \beta Y=\beta X \times Y$. Then by Proposition 3.1 of [2],

$$
\mathscr{P}(X \times Y)=\mathscr{P} X \times \mathscr{P} Y=\mathscr{P} X \times Y=\beta X \times Y \quad\left(\text { as } X \in \mathscr{P}^{\prime}\right)=\beta(X \times Y),
$$

i.e., $X \times Y \in \mathscr{P}^{\prime}$. The condition that $\mathbf{R} \in \mathcal{P}$ is not necessary for the above result. If $\mathscr{P}$ is the class of compact spaces then $\mathbf{R} \notin \mathscr{P}$, but since $\mathcal{P}^{\prime}$ consists of 
all Tychonoff spaces, $\mathscr{P}^{\prime}$ satisfies the condition that if $X \in \mathscr{P}^{\prime}$ and $Y$ is compact, then $X \times Y \in \mathcal{P}^{\prime}$.

3. 0-dimensional pseudocompactness properties. If $X$ is 0 -dimensional (i.e., has a base of clopen sets), let $\beta_{0} X$ denote the Banaschewski maximal 0 dimensional compactification of $X$ (see [1]). The space $\beta_{0} X$ is the Stone space (maximal ideal space) of the Boolean algebra of clopen subsets of $X$. If $\mathscr{P}_{0}$ is a class of 0 -dimensional spaces which contains $\{0,1\}$, is closed hereditary and preserved under the formation of products (an epireflective, almost fitting subcategory of the category of 0 -dimensional spaces), then $\mathscr{P}_{0}$ is called a 0 dimensional extension property. If $X$ is 0 -dimensional and $\mathscr{P}_{0}$ is a 0 -dimensional extension property, then there is a space $\mathscr{P}_{0} X$ such that $X \subseteq \mathscr{P}_{0} X \subseteq \beta_{0} X$, $\mathscr{P}_{0} X \in \mathscr{P}_{0}$, and any continuous map from $X$ to a space in $\mathscr{P}_{0}$ admits a continuous extension to $\mathscr{P}_{0} X$. This space can be produced in a way analogous to the construction of $\mathscr{P} X$ in [5]. The concept of $\mathscr{P}_{0}$-pseudocompactness is analogous to that of $\mathcal{P}$-pseudocompactness defined above. Since $N \times \beta D$ is 0 dimensional, in view of Example 2.2 above, question one asked in the introduction has a negative answer when applied to 0-dimensional extension properties. However, Example 2.1 above will not work in the 0-dimensional case of question two. The following example shows that the answer to question two with respect to 0 -dimensional extension properties is negative.

3.1. EXAMPLE. Let $\omega_{1}^{*}=\omega_{1} \cup\left\{\omega_{1}\right\}$ denote the one-point compactification of $\omega_{1}$, the totally ordered space of countable ordinals. Let $\mathscr{P}_{0}$ be the class of all 0 dimensional, $\boldsymbol{\aleph}_{0}$-bounded spaces. Then $X=N \times \omega_{1}^{*} \in \mathscr{P}_{0}^{\prime}$ (if $p \in \beta_{0} X$ and $p$ is not in the closure of $N \times\left\{\omega_{1}\right\}$, then $p$ is in the closure of a complement of a neighborhood of $N \times\left\{\omega_{1}\right\}$ which must be countable). For each $i \in N$ let $f_{i}$ be a bijection between $D$, the discrete space of cardinality $\boldsymbol{\kappa}_{1}$, and the isolated points of $\omega_{1}^{*}$, and let $f_{i}^{*}: \beta D \rightarrow \omega_{1}^{*}$ be the Stone extension of $f_{i}$. Then $f_{i}^{*}$ is perfect and irreducible (i.e., no proper closed subset of $\beta D$ maps onto $\omega_{1}^{*}$ ). Thus $\cup_{i \in N} f_{i}: N \times \beta D \rightarrow N \times \omega_{1}^{*}$ is perfect and irreducible. By Lemma 2.14 of [8], $N \times \beta D=E\left(N \times \omega_{1}^{*}\right)$. However, Example 2.2 above shows that $N \times \beta D \notin \mathcal{P}_{0}^{\prime}$ (we showed $N \times \beta D \notin \mathcal{P}^{\prime}$ where $\mathcal{P}$ is the class of $\kappa_{0}$-bounded spaces, but since $N \times \beta D$ is extremally disconnected we have $\beta_{0}(N \times \beta D)$ $=\beta(N \times \beta D)$ and, hence, $\mathscr{P}_{0}(N \times \beta D)=\mathscr{P}(N \times \beta D) \varsubsetneqq \beta(N \times \beta D)$ by Example 2.2).

We can sharpen the result of Theorem 2.3 when restricting ourselves to 0 dimensional extension properties.

3.2 THEOREM. Let $\mathscr{P}_{0}$ be a 0-dimensional extension property not all of whose members are pseudocompact. Let $X \in \mathscr{P}_{0}^{\prime}$ and let $Y$ be compact and 0 dimensional. Then $X \times Y \in \mathscr{\rho}_{0}^{\prime}$.

Proof. Since not every member of $\mathscr{P}_{0}$ is pseudocompact it follows that some members of $\mathscr{P}_{0}$ contain a $C$-embedded, hence closed, copy of $N$ (see 1.21 and 3B3 of [4]). Thus $N \in \mathscr{P}_{0}$. By Theorem 2.2 of [9], every member of $\mathscr{P}_{0}^{\prime}$ is pseudocompact. 
Let $X \in \mathscr{P}_{0}^{\prime}$ and $Y$ be compact and 0 -dimensional. Then $X \times Y$ is pseudocompact. Hence by Theorem 2.2 of [2],

$$
\beta_{0}(X \times Y)=\beta_{0} X \times \beta_{0} Y=\beta_{0} X \times Y .
$$

Then by Proposition 3.1 of [2],

$$
\mathscr{P}_{0}(X \times Y)=\mathscr{P}_{0} X \times \mathscr{P}_{0} Y=\mathscr{P}_{0} X \times Y=\beta_{0} X \times Y=\beta_{0}(X \times Y),
$$

hence $X \times Y \in \mathscr{P}_{0}^{\prime}$.

Corollary 2.10 of [9] shows that if $\mathscr{P}_{0}$ is a 0 -dimensional extension property, then either $\mathscr{P}_{0}$ is the class of compact 0 -dimensional spaces or $\mathscr{P}_{0}^{\prime}$ does not properly contain the class of pseudocompact 0 -dimensional spaces. The question is then raised of whether or not the corresponding statement for (not necessarily 0 -dimensional) extension properties holds. If $\mathscr{P}$ is the class of $I \times$ $N$-compact spaces (if $E$ is a space, then $X$ is $E$-compact if $X$ can be embedded as a closed subset of $E^{m}$ for some cardinal number $m$; see [6]), then $\mathcal{P}$ is an extension property. But clearly $\mathcal{P}$ contains noncompact spaces (e.g., $N \in \mathscr{P}$ ). Also $\mathscr{P}^{\prime}$ properly contains the class of pseudocompact spaces. This follows from 2.2(e) of [9] and the fact that $\mathbf{R} \in \mathcal{P}^{\prime}$, and $\mathbf{R}$ is not pseudocompact. Thus the statement for arbitrary extension properties corresponding to 2.10 of [9] is not valid.

\section{REFERENCES}

1. B. Banaschewski, Über nulldimensionale Räume, Math. Nachr. 13 (1955), 129-140. MR 19, 157.

2. S. Broverman, The topological extension of a product, Canad. Math. Bull. (to appear).

3. Z. Frolik, The topological product of two pseudo-compact spaces, Czechoslovak Math. J. 10 (85) (1960), 339-349. MR 22 \#7099.

4. L. Gillman and M. Jerison, Rings of continuous functions, Univ. Ser. in Higher Math., Van Nostrand, Princeton, N.J., 1960. MR 22 \#6994.

5. H. Herrlich and J. Van der Slot, Properties which are closely related to compactness, Nederl. Akad. Wetensch. Proc. Ser. A 70 = Indag. Math. 29 (1967), 524-529. MR 36 \#898.

6. S. Mrówka, Further results on E-compact spaces. I, Acta Math. 120 (1968), 161-185. MR 37 \#2165.

7. R. G. Woods, Some $\aleph_{0}$-bounded subsets of Stone-Čech compactifications, Israel J. Math. 9 (1971), 250-256. MR 43 \#3997.

8. , Ideals of pseudocompact regular closed sets and absolutes of Hewitt realcompactifications, General Topology and Appl. 2 (1972), 315-331. MR 47 \# 7698.

9. _- Topological extension properties, Trans. Amer. Math. Soc. 210 (1975), 365-385.

Department of Mathematics, University of Manitoba, Winnipeg, Manitoba, Canada R3T 2N2 